\title{
The potential role of suppressive therapy for sex partners in the prevention of neonatal herpes: a health economic analysis
}

\author{
R V Barnabas, H Carabin, G P Garnett
}

See end of article for authors' affiliations

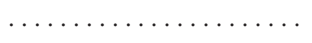

Correspondence to: G P Garnett, Department Infectious Disease

Epidemiology, Faculty of Medicine, Imperial

College, St Mary's, Norfolk

Place, London W2 1PG,

UK; g.garnett@ic.ac.uk

Accepted for publication 20 June 2002

\begin{abstract}
Background: The development of suppressive therapy and type specific tests for herpes infections allow for screening to reduce the risk of neonatal herpes.

Objectives: To assess the potential effectiveness, cost effectiveness, and benefit of suppressive therapy among herpes simplex virus serodiscordant sex partners during pregnancy.

Methods: Decision and economic analyses are used to compare the incidence and costs of neonatal herpes in California (2000) for three interventions: (1) no management; (2) current guidelines (caesarean delivery for women with lesions); (3) screening for women at risk and use of suppressive treatment in sex partners.

Results: Screening and suppressive therapy are the most effective interventions, while current guidelines have limited effectiveness, but the latter provide the most cost effective results.

Conclusions: While current guidelines are cost saving, they forgo a potential $82 \%$ decrease in neonatal herpes incidence that would be possible with screening and suppressive therapy if society were willing to pay the necessary US\$363000 per case prevented. To evaluate HSV screening and drug therapy completely, clinical trials and an economic assessment of infant mortality "value" to society are required.
\end{abstract}

$\mathrm{N}$ eonatal herpes is largely the result of mother to child transmission of the herpes simplex virus (HSV) during labour and delivery. It is the most serious direct consequence of genital herpes, ${ }^{1}$ a chronic infection with no cure available. Women who have primary HSV infection late in pregnancy are estimated to have a $45 \%$ risk of infecting their infants, much higher than the $<1 \%$ risk associated with secondary infection. ${ }^{2}{ }^{3}$ It follows that neonatal herpes primary prevention efforts should focus on preventing incident infections in pregnant women. Type specific HSV tests can identify women at risk of incident infection ${ }^{4}$ : theoretically, suppressive antiviral therapy can decrease viral shedding in their sex partners and potentially decrease horizontal HSV transmission. Decision and economic analyses can help quantify the possible effectiveness and cost effectiveness of such a strategy and identify research priorities. ${ }^{56}$

\section{BACKGROUND}

Neonatal herpes has the highest case fatality "rate" of any neonatal infection, more than $60 \%$ in untreated cases, ${ }^{7}$ but therapy reduces this to $20 \%{ }^{8}$ Even with antivirals long term neurological sequelae are still seen in almost a quarter of cases. ${ }^{79}$ A study that looked at Californian hospital discharge data in 1985, 1990, and 1995 found, respectively, 11.7, 11.3, and 11.4/100 000 live births had a diagnosis of HSV ${ }^{10}$ Recent neonatal herpes incidence estimates range between 20/ 100000 and 50/100 000 live births ${ }^{2}$ with 40/100 000 live births reported at the University of Washington. ${ }^{5}$

Genital herpes is usually caused by herpes simplex virus type 2 (HSV-2), but increasingly primary infections are caused by herpes simplex type 1 (HSV-1). ${ }^{8}$ In the United States, where HSV-2 population seroprevalence was estimated to be $22 \%,{ }^{11}$ $70 \%$ of neonatal herpes is caused by HSV-2. ${ }^{8}$ Eighty per cent of genital herpes is asymptomatic or unrecognised ${ }^{1}$ and HSV-2 seropositive people shed virus from the genital tract on about $3 \%$ of days, ${ }^{12}$ producing a reservoir for HSV spread. Chronic aciclovir therapy reduces the frequency of HSV DNA detection by a median of $80 \%,{ }^{13}$ presumably through a decrease in viral shedding. It follows that men in serodiscordant couples (identified through type specific tests) could be treated to suppress asymptomatic shedding and reduce HSV transmission to susceptible women.

Debates regarding the role of HSV-2 specific serology in routine antenatal care have raised important questions. ${ }^{56}$ Whether aciclovir therapy reduces HSV transmission is currently being addressed in a clinical trial. ${ }^{14}$ The effectiveness and costs of screening and subsequent intervention (abstinence, condom use, or suppressive aciclovir) requires evaluation. This theoretical paper considers the epidemiological and economic outcome of identifying serodiscordant couples and using prophylactic aciclovir as a control strategy for neonatal herpes.

\section{METHODS}

Three programmes to decrease mother to child transmission of HSV are compared from a societal viewpoint to assess net costs versus consequences ${ }^{15}$ : The first programme $(\mathrm{Pl})$ is "no management" which has no costs above those of a normal pregnancy, but has the consequences of neonatal herpes. The second programme (P2) recommends caesarean section for women presenting with genital lesions at delivery. ${ }^{16}$ Programme 3 (P3), treating partners with aciclovir, counsels, and screens all pregnant women and, if necessary, their partners who receive aciclovir to reduce transmission of HSV. P3 includes counselling regarding condom usage and risks of oral $\operatorname{sex}^{17}$ and caesarean delivery for women with herpetic lesions at the time of delivery. We first construct a decision analysis model, including the three policies, which estimates the incidence of vertical transmission from pregnant women over the course of the year 2000, in California. We then estimate costs and consequences of each programme to calculate the cost per case averted and cost benefit ratio for the interventions compared with the "no management" alternative. The methods used are described in detail in appendix A on the STI website. 


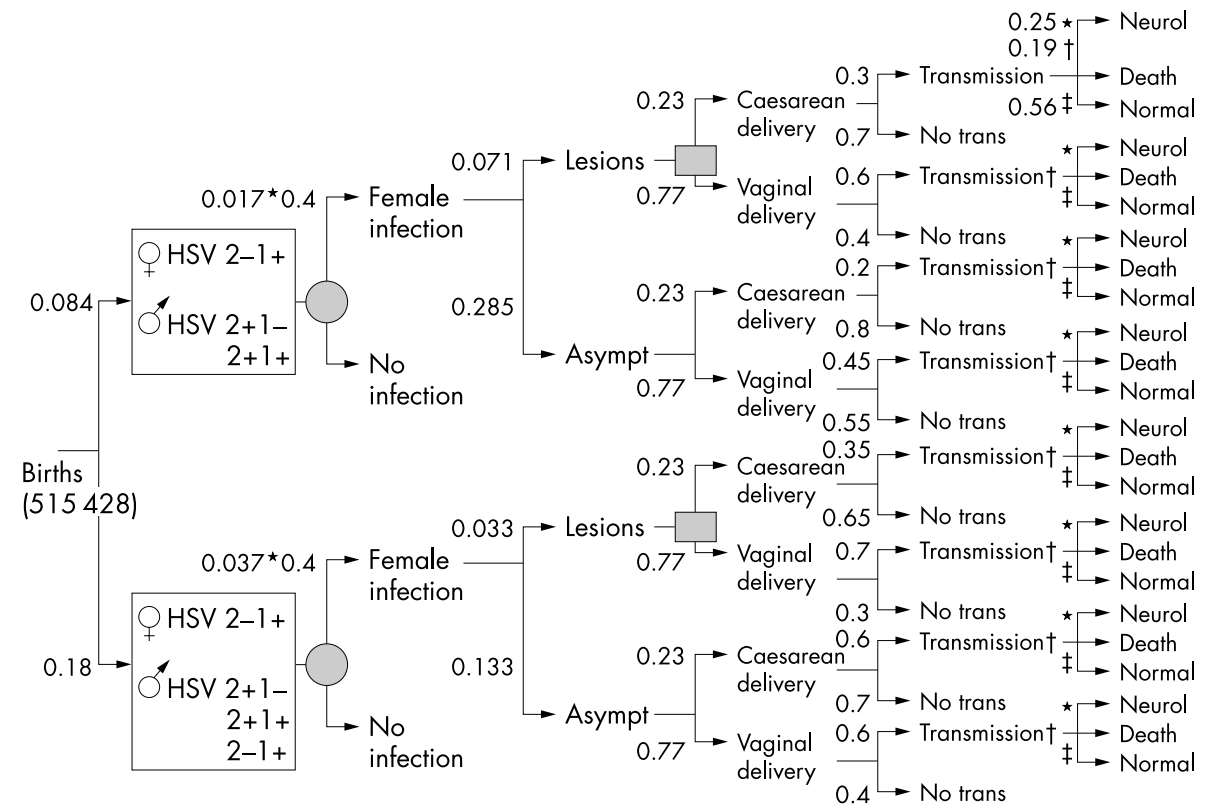

Figure 1 Decision analysis model representing primary and non-primary first episode HSV infection among pregnant women in California in 2000 and consequent mother to child transmission of HSV. The decision analysis model shows the chance of incident HSV infection among women in the third trimester of pregnancy in California in 2000 and the subsequent chance of neonatal herpes. Women infected in the third trimester were calculated by the product of incident HSV infection in pregnancy and the fraction that were infected in the third trimester. ${ }^{3}$ Asympt = asymptomatic shedding; No trans = no transmission; Neurol = neurological disability. Cases of neonatal herpes were classified as neurological disability, death, or normal (indicating no long term complications of HSV infection). Probability of event: ${ }^{\star} 0.25 ; \dagger 0.19 ; \neq 0.56$. Intervention P2 (shaded squares) = increased caesarean sections with lesions (to 85\%). Intervention P3 (shaded circles) = suppression decreases transmission (by $80 \%$ ).

\section{Epidemiological model-decision analysis}

A theoretical transmission model is used to calculate the number of neonatal infections as a result of primary or non-primary first episode HSV infection among women late in pregnancy (fig 1). The observed rate of transmission from partner to mother and then to the child is used to estimate the number of neonatal infections and is largely based on a study by Brown and colleagues. ${ }^{3}$ ACOG guidelines (P2) increase the likelihood of caesarean delivery from $23 \%$ to $85 \%{ }^{18}$ and decrease vertical HSV transmission by $50 \%{ }^{19}$ The use of counselling and suppressive therapy (P3) is assumed to reduce the risk of partner to mother transmission by $80 \%{ }^{13}$

For example, it was estimated that $8.4 \%$ of couples consist of HSV-1 positive and HSV-2 negative women with HSV-2 positive men. ${ }^{311}$ We assume that $1.7 \%$ (95\% CI $1.1 \%$ to $2.3 \%$ ) of these women will seroconvert during pregnancy, but only $40 \%$ of those seroconversions will occur in the third trimester. ${ }^{3}$ Of those who acquire HSV-2 it was estimated that $28 \%$ shed HSV asymptomatically at the time of delivery. Without intervention, $77 \%$ of those women will have vaginal deliveries, ${ }^{18}$ and
$45 \%$ of their infants would become infected. Long term, $56 \%$ of HSV positive infants would be normal, 19\% would die, and $25 \%$ would have long term neurological disability. ${ }^{78}$

Because of the limited information we had to make several assumptions, itemised in table 1. Assuming monogamy in the third trimester of pregnancy, assuming that partnerships are independent of HSV status, assuming $100 \%$ participation, a $>99 \%$ diagnostic sensitivity and specificity and an $80 \%$ reduced transmission with suppressive therapy all increase the impact of screening and therapy. Concomitantly, we favour programme 2 by assuming a scaled up version of current guidelines for education and surveillance, that the dissemination of ACOG guidelines increases caesarean sections indicated by genital lesions (85\%), ${ }^{18}$ and that the effectiveness of elective caesarean section is $50 \%$. However, evidence for caesarean section efficacy is limited. ${ }^{19}$

\section{Costs}

Costs are calculated from a societal perspective using the human capital approach for maternal mortality and long term

Table 1 Assumptions and limitations considered in the sensitivity and uncertainty analysis

\begin{tabular}{|c|c|c|}
\hline Assumptions and limitations & Analysis range (\%) & Uncertainty analysis distribution \\
\hline \multicolumn{3}{|l|}{ Assumptions maximising $\S P 3$ effectiveness } \\
\hline Monogamy in the 3rd trimester & - & - \\
\hline Partnerships independent of HSV serostatus & $22-26 \dagger$ & Uniform \\
\hline Participation $100 \%$ & $60-100 *$ & \\
\hline Diagnostic sensitivity and specificity $>99 \%$ & - & - \\
\hline Aciclovir effectiveness $=80 \%$ & 50-80-90† & Triangular \\
\hline \multicolumn{3}{|c|}{ Other factors varied in sensitivity and uncertainty analysis } \\
\hline Caesarean section effectiveness $=50 \%$ & 40-50-90† & Triangular \\
\hline Cost of treating acute neonatal herpes & US\$1500-26196-50000‡ & Triangular \\
\hline Cost of diagnostic kit & US\$16-70-100‡ & Triangular \\
\hline Discount rate & $0.01-0.07^{*}$ & \\
\hline
\end{tabular}

${ }^{*}$ Considered in univariate sensitivity analysis. †Considered in multivariate uncertainty analysis. $¥$ Considered in both univariate and multivariate analysis. $\S \mathrm{P3}=$ aciclovir therapy for partners to suppress asymptomatic shedding of HSV. 


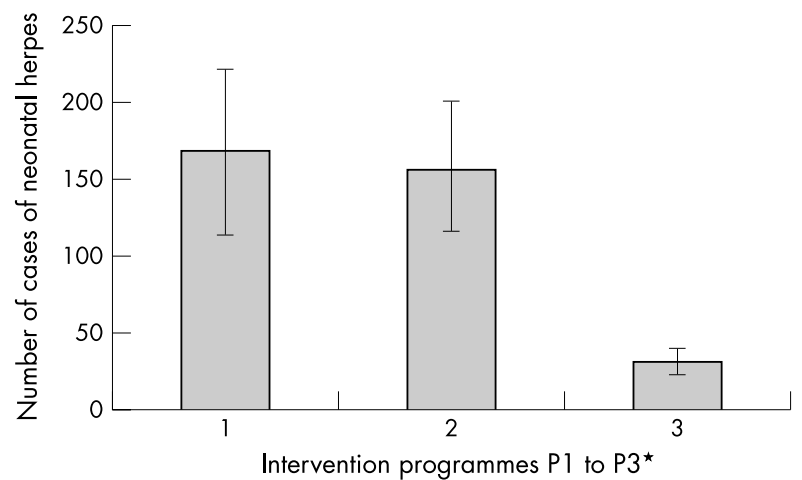

Figure 2 Number of cases of neonatal herpes for P1 to P3* for serodiscordant couples. ${ }^{\star} \mathrm{Pl}=$ "Do nothing" programme. P2 = performing caesarean sections for women who present with genital lesions at the time of delivery. P3 = aciclovir therapy for partners to suppress asymptomatic shedding of HSV. Error bars indicate $95 \%$ $\mathrm{Cl}$

neurological sequelae in infants. ${ }^{15}$ The human capital approach uses estimates of lost wages to evaluate morbidity and mortality. Our analysis fails to take account of neonatal deaths, which would normally be assessed in a cost utility analysis or using a willingness to pay approach. To bring past estimates of costs up to year 2000 values we used the consumer price index (CPI), and, in calculating the value of future costs we used a 3\% discount rate. ${ }^{15}$

Hospital and personnel costs for both interventions and outcomes were obtained from the Californian Department of Health, ${ }^{20}$ and the US Department of Labour. ${ }^{21}$ Economic indicators used were from the Californian Department of Finance. ${ }^{22}$ The cost of acute neonatal herpes (US\$26 196 per case for the first year) ) $^{23}$ and long term disability (US\$782 035 per case until age 15 years) $)^{24}$ were estimated from the literature. The itemised cost of consequence menu (table Al) is found in appendix A on the STI website.

To implement ACOG guidelines an obstetrician would disseminate written information. Costs were included for the additional caesarean sections indicated by genital lesions (surgical complications, longer hospital stay, and increased maternal mortality rate). For screening and aciclovir prophylaxis, two full time obstetricians would run seminars for obstetricians covering type specific HSV diagnostics and suppressive therapy. For both programmes, two nurses would provide follow up to ensure programme compliance and an epidemiologist, assisted by a data clerk, would coordinate monitoring and feedback. The cost of a point of care, HSV type specific diagnostic test was estimated to be US\$70 per couple per pregnancy. ${ }^{25}$ Cost menus for the programmes, implemented over 1 year are provided in appendix A on the STI website. The cost benefit ratio is the saving/cost ratio and was calculated as the benefit for every US\$1 spent in each programme.

Univariate sensitivity analysis and multivariate "uncertainty" analysis were used to explore the influence of particular variables and the overall credibility of results (table 1).

\section{RESULTS}

The estimated yearly number of neonatal herpes cases for the no management approach (P1) is 169 (fig 2). Screening and suppressive therapy (P3) would reduce this number to 31 (an $82 \%$ decrease with $95 \%$ CI $73 \%$ to $87 \%$ ). With ACOG guidelines (P2), the number of cases would be reduced to 155 (an $8 \%, 95 \%$ CI -1\% to $26 \%$ ), but the confidence interval overlaps with Pl. Screening and therapy prevents $80 \%$ (95\% CI $71 \%$ to $86 \%$ ) of cases compared to ACOG guidelines.

The costs and consequences of the scenarios are summarised in table 2. The most expensive alternative was suppressive aciclovir for partners, at just over US $\$ 58$ million, a cost effectiveness ratio of US\$362942 per case averted or US\$29 178 per life year saved. Caesarean section for women with genital lesions, is substantially less costly at US\$613 527 with a cost of US\$3096 per life year saved. Looking more closely at individual components, screening makes up most of P3's economic costs (85\%). The cost of counselling alone is over US\$10 million. Even though the minimum number of staff necessary was considered, favouring the interventions, personnel still constitute the bulk of costs.

The costs were used to calculate the saving/cost ratio (or cost benefit ratio) which, when comparing two programmes, is the difference between the cost of their consequences divided by the difference between costs of implementation (table 3). For every US\$ spent, the saving for implementing P2 is US\$5.80 compared to no specific management (P1). For P3, more is spent on the intervention programme than is saved, with US\$0.62 saved for US\$ spent. Similarly, the savings of implementing P3 do not outweigh the extra costs compared to $\mathrm{P} 2$. The incremental cost effectiveness for $\mathrm{P} 3$ versus $\mathrm{P} 1$ and $\mathrm{P} 3$ versus P2 were US\$103580 and US\$143648 per case prevented respectively.

The sensitivity of the saving/cost ratio to uncertain variables was assessed (table 1). Univariate analysis of acute neonatal herpes treatment, aciclovir and diagnostic costs, discount rate (fig Bl on STI website), and participation did not make

Table 2 Comparison of total costs (US\$) of P1 to P2 and P3 * in California in 2000

\begin{tabular}{|c|c|c|c|}
\hline & $\mathrm{P} 1^{*}$ (US\$) & P2* (US\$) & P3* (US\$) \\
\hline \multicolumn{4}{|c|}{ Implementation costs (US\$) } \\
\hline Financial & - & 110528 & 42189275 \\
\hline Opportunity & - & 502999 & 15957443 \\
\hline Economic & - & 613528 & 58146718 \\
\hline \multicolumn{4}{|c|}{ Total costs associated with: } \\
\hline Training & - & 101463 & 1030554 \\
\hline Screening & - & 0 & 49540509 \\
\hline Treatment & - & 502999 & 8041487 \\
\hline Surveillance & - & 9065 & 8063 \\
\hline \multicolumn{4}{|c|}{ Cost of the consequences (US\$) } \\
\hline Financial & 21948876 & 20167699 & 4033540 \\
\hline Opportunity & 21903130 & 20125665 & 4025133 \\
\hline Economic & 43852006 & 40293364 & 8058673 \\
\hline
\end{tabular}

*P1 = "no specific management" programme. P2 = performing caesarean sections for women who present with genital lesions at the time of delivery. P3 = aciclovir therapy for partners to suppress asymptomatic shedding of HSV. Financial cost is the value of the resources consumed or saved by the programme. Opportunity cost is the forgone benefits because the resource is not available for its best alternative use. Economic cost is the sum of the financial and opportunity cost. ${ }^{15}$ 
Table 3 Saving/cost ratio (or the cost benefit ratio) comparing neonatal herpes intervention programmes $\mathrm{Pl}$ to P3* in California in 2000. The saving is shown for every US\$ spent

\begin{tabular}{ll}
\hline Comparison of programmes & $\begin{array}{l}\text { Saving in US\$ for every US\$ } \\
\text { spent }\end{array}$ \\
\hline P1 v P2 & 5.80 \\
P1 v P3 & 0.62 \\
P2 v P3 & 0.56
\end{tabular}

*P1 = "Do nothing" programme. $\mathrm{P} 2$ = performing caesarean sections for women who present with genital lesions at the time of delivery. P3 = aciclovir therapy for partners to suppress asymptomatic shedding of HSV

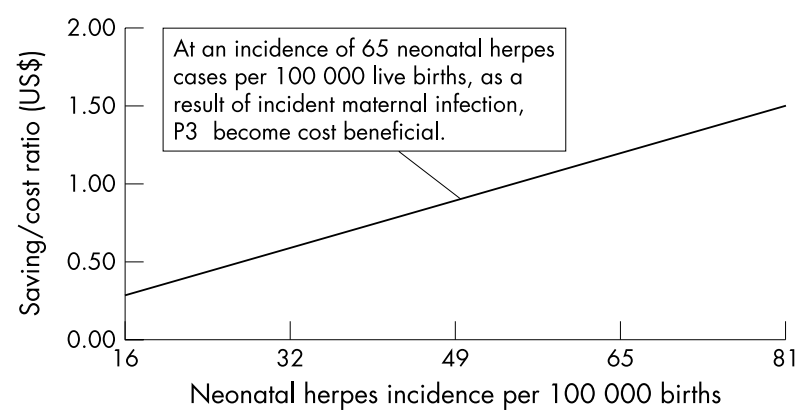

Figure 3 The effects of varying the incidence of neonatal herpes (per 100000 births) on the saving/cost ratio (the saving for every US $\$$ spent) for $\mathrm{P} 3^{*} \vee \mathrm{P1}$. *P1 = "Do nothing" programme. $\mathrm{P} 2=$ performing caesarean sections for women who present with genital lesions at the time of delivery. P3 = aciclovir therapy for partners to suppress asymptomatic shedding of HSV.

screening cost beneficial. Bivariate analysis of the diagnostic and drugs costs showed that the best saving/cost ratios were for the lowest prices, but the fixed costs (personnel, hospital costs, and capital items) always prevented savings from outweighing costs. Decreasing the participation increased the cost per HSV case averted. With multivariate uncertainty analysis (parameters detailed in table 1) the qualitative results do not change and $\mathrm{P} 3$ is not cost beneficial compared to P1.

Regression shows that the number of serodiscordant couples in the population has the largest influence on the number of neonatal HSV cases and the efficacy of aciclovir is the most influential factor in determining P3 effectiveness, but within the ranges considered $\mathrm{P} 3$ was not cost saving compared to P1. Because neonatal herpes incidence is uncertain, we explored the impact of varying this incidence under the general assumptions of the model. An incidence of 65 neonatal cases per 100000 births as a result of maternal incident infection would make P3 a cost beneficial intervention (fig 3).

\section{DISCUSSION}

Our cost benefit analysis compares alternative programmes to prevent neonatal HSV in pregnant women. The decision analysis showed that treating partners of those women at risk for acquiring HSV late in pregnancy (P3) had the greatest impact on neonatal herpes when compared to no specific management (P1) and current guidelines (P2). P3 offers the best reduction in neonatal herpes incidence and mortality but its costs outweighed the benefits when compared to no management.

Rouse and Stringer also found that screening for HSV serodiscordant couples in pregnancy was not cost effective, but the intervention considered by that study was counselling regarding risky behaviour. ${ }^{26}$ In the United Kingdom and Australia where neonatal herpes is rare compared to the United States,
Qutub and Mindel, respectively, found that antenatal HSV screening was not cost effective. ${ }^{27} 28$

In a previous neonatal herpes study, Randolph and colleagues found caesarean sections to decrease neonatal herpes incidence had a good cost utility value for women with first episode genital herpes, saving US\$2600 per quality adjusted life year (QALY, the number of maternal life years lost is subtracted from the gain in life years for neonatal cases of HSV averted) but not for recurrent disease (costing US\$203000 per QALY). ${ }^{29}$ Scott and colleagues demonstrated a reduction in the number of caesarean sections necessary among those women on prophylactic aciclovir after first episode genital herpes. ${ }^{30}$ While we did not consider aciclovir prophylaxis in women with first episode herpes we did find that aciclovir prophylaxis for HSV-2 seropositive men decreased the predicted number of caesarean sections among their susceptible partners.

When using analytical tools in decision making it is important to note the influence of assumptions. Despite most of our assumptions favouring screening, it was not cost beneficial. However, this finding must be treated with caution because the "value" of neonatal mortality, an important component was not accounted for in the economic analysis. The obvious difficulty with the human capital approach, which ascribes a nil value to neonatal mortality, is that it disregards the psychological impact of the death of a newborn child for the family. If society were "willing to pay" US\$1 million per death averted, P3 would be cost beneficial compared to P1 and US $\$ 1.1$ million would make P3 cost beneficial compared to P2. Economic techniques that equitably value a healthy newborn child's life, in a manner that reflects the worth to society, could tip the scales and intensive programmes that involve screening at a population level may prove to be cost beneficial.

Estimates of society's willingness to pay are controversial but could provide some insights, as could improved utility measures, which take into account of the impact of neonatal death or exposure to the risk of such deaths caused by a sexually transmitted infection. Random sample population surveys containing relevant detail would be required to create such measures.

Ideally, a cost benefit analysis would tell us whether a programme is worth implementing - that is, whether the costs outweigh the benefits. But with a crucial piece of data missing, the cost of neonatal mortality, the most useful economic measure is probably the cost per HSV case averted (US\$363 000) rather than the saving/cost ratio. This figure will have to be balanced against other demands on health resources. Crucially, the economic study showed how the fixed costs of personnel prevented the proposed intervention strategy from becoming cost effective despite the marked gains in healthy infants. It is challenging to consider how intervention programmes can be structured differently to make them more cost effective, perhaps through employing nurse practitioners rather than physicians where possible.

In addition to neonatal mortality costs, the regression analysis showed that aciclovir efficacy and the number of HSV serodiscordant partners are key in determining whether screening and suppressive therapy is cost effective. Aciclovir is being evaluated in a clinical trial. ${ }^{14}$ However, neonatal HSV incidence, as a result of first episode maternal infection, still needs to be measured. Programme 3 becomes cost beneficial at an incidence of 65/100 000 live births, a conceivable rate. Neonatal herpes is not a notifiable disease with nonpathognomonic signs and symptoms which may lead to under-reporting of cases. ${ }^{731}$ The incidence obtained in this study for neonatal herpes resulting from maternal first episode HSV infection without intervention is 32/100 000 live births, using transmission probabilities from the Brown study $^{3}$ (the sample size of which is probably too small to give an accurate estimate of neonatal herpes incidence). Improved surveillance of herpes epidemiology would contribute to the robustness of future analyses of prevention programmes. 


\section{Key messages}

(1) We need to think about innovative prevention strategies for the serious complications of chronic viral STIs, such as HSV and HIV. In the case of vertical HSV transmission new diagnostic tests and suppressive aciclovir therapy could make it possible to screen for women at risk during pregnancy and treat their partners to prevent horizontal and, consequently, mother to child HSV transmission. This intervention requires quantitative evaluation of the potential efficacy and costs and benefits to society.

(2) Screening and aciclovir suppression has the potential to decrease neonatal herpes incidence by $82 \%$ but we have to ask if we are willing to pay US $\$ 363000$ per case prevented to implement this HSV control strategy, bearing in mind that the "effective" decrease in neonatal herpes may be less.

(3) The study identifies three factors that will determine whether screening and therapy is cost beneficial: (a) the incidence of neonatal herpes, (b) the efficacy of aciclovir in decreasing the transmission, and (c) the "value" of neonatal mortality.

(4) The study draws attention to the high cost of personnel in intensive intervention programmes, challenging us to bear this in mind when designing health interventions.

While ACOG guidelines are intended to prevent cases of neonatal herpes resulting from recurrent and first episode maternal infection, ${ }^{16}$ the small effectiveness of the current guidelines (P2) among women with incident HSV could bring the value of this strategy into question. The recommendation of antiviral treatment for primary or non-primary first episode herpes without screening means that $80 \%$ of genital herpes cases are untreated. Unless potential cases are identified through screening, further interventions of any sort, including counselling, will not be possible.

Accurate valuation of neonatal mortality, accurate data on neonatal herpes incidence, lower diagnostic and aciclovir costs, and clinical studies on aciclovir efficacy may mean that screening and suppressive therapy could be cost beneficial. Before undertaking a widespread screening programme the risks of psychosexual problems associated with a diagnosis of genital herpes, especially among serodiscordant couples, must be considered and adequate provision made for counselling. ${ }^{6}$ In addition to the need for epidemiological and economic data, policy planners must assess the acceptability of such strategies to pregnant women and their partners.

\section{CONTRIBUTORS}

GG contributed to the design of the study and the epidemiological model; RB constructed the epidemiological model and carried out the health economic analysis; and HC advised on and contributed to the health economic analysis. The manuscript was drafted in a collaboration between the authors.

\section{ACKNOWLEDGEMENTS}

Ruanne Barnabas would like to thank the Rhodes Trust, Hélène Carabin the Wellcome Trust, and Geoff Garnett the Royal Society for grant support.

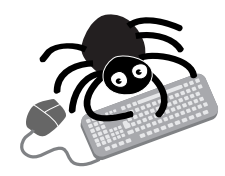

Appendices containing three tables and a figure are available on the STI website.

\section{Authors' affiliations}

R V Barnabas, Nuffield Department of Medicine, University of Oxford, Oxford, UK
R V Barnabas, H Carabin, G P Garnett, Department of Infectious Disease Epidemiology, Faculty of Medicine, Imperial College, St Mary's, London, UK

\section{REFERENCES}

1 Corey L, Handsfield $\mathrm{HH}$. Genital herpes and public health: addressing a global problem. JAMA 2000;283:791-4.

2 Smith JR, Cowan FM, Munday P. The management of herpes simplex virus infection in pregnancy. Br J Obstet Gynaecol 1998; 105:255-60.

3 Brown ZA, Selke S, Zeh J, et al. The acquisition of herpes simplex virus during pregnancy. N Engl J Med 1997;337:509-15.

4 Ashley RL, Wald A. Genital herpes: review of the epidemic and potential use of type-specific serology. Clin Microbiol Rev 1999;12:1-8.

5 Brown ZA. HSV-2 soecific serology should be offered routinely to antenatal patients. Rev Med Virol 2000;10:141-4.

6 Wilkinson D, Barton W, Cowan F. HSV-2 specific serology should not be offered routinely to antenatal patients. Rev Med Virol 2000; 10:145-53

7 Scott LL. Prevention of perinatal herpes: prophylactic antiviral therapy? Clin Obstet Gynecol 1999:42:134-48; quiz 174-5.

8 Marques AR, Straus SE. Herpes simplex type 2 infections-an update. Adv Intern Med 2000;45:175-208

9 Kimberlin DW, Lin CY, Jacobs RF, et al. Natural history of neonatal herpes simplex virus infections in the acyclovir era. Pediatrics 2001:108:223-9.

10 Gutierrez KM, Falkovitz Halpern MS, Maldonado Y, et al. The epidemiology of neonatal herpes simplex virus infections in California from 1985 to 1995. J Infect Dis 1999; 180:199-202.

11 Fleming DT, McQuillan GM, Johnson RE, et al. Herpes simplex virus type 2 in the United States, 1976 to 1994. N Engl J Med 1997;337:1105-11.

12 Wald A, Zeh J, Selke S, et al. Reactivation of genital herpes simplex virus type 2 infection in asymptomatic seropositive persons. N Engl J Med 2000;342:844-50.

13 Wald A, Corey L, Cone R, et al. Frequent genital herpes simplex virus 2 shedding in immunocompetent women. Effect of acyclovir treatment. $J$ Clin Invest 1997;99:1092-7.

14 Wald A, Corey L. Antiviral therapies for long-term suppression of genital herpes. Jama 1999:281:1169-70; discussion 1170-1.

15 Drummond $\mathbf{M}$. Methods for the economic evaluation of health care programmes. 2nd ed. Oxford: Oxford University Press, 1997.

16 ACOG Practice Bulletin. Management of herpes in pregnancy. Number 8 October 1999. Clinical management guidelines for obstetrician-gynecologists. Int J Gynaecol Obstet 2000;68: 165-73.

17 Clinical Effectiveness Group (Association of Genitourinary Medicine and the Medical Society for the Study of Venereal Diseases). National guideline for the management of genital herpes. Sex Transm Infect 1999;75(Suppl 1):S24-8.

18 Scott LL, Alexander J. Cost-effectiveness of acyclovir suppression to prevent recurrent genital herpes in term pregnancy. Am J Perinatol 1998; 15:57-62.

19 Brown ZA, Benedetti J, Ashley R, et al. Neonatal herpes simplex virus infection in relation to asymptomatic maternal infection at the time of labor. N Engl J Med 1991;324:1247-52.

20 HCUPnet. California State Inpatient Database. In: HCUP, 1997

21 Labour UDo. Occupational outlook handbook. 2000-1. Bureau of Labour Statistics, 1997.

22 Finance CDo. Economic indicators May-June 2000. California: Department of Finance, June 2000.

23 Mennemeyer ST, Cyr LP, Whitley RJ. Antiviral therapy for neonatal herpes simplex virus: a cost-effectiveness analysis. Am J Manag Care 1997; 3:1551-8.

24 Waitzman NJ, ed. Economic costs of birth defects and cerebral palsy-United States. Morbidity and Mortality Weekly Report 1995;44:694-9

25 Centers for Disease Control and Prevention. Report of the Genital Herpes Prevention Consultants Meeting. In: Centers for Disease Contro and Prevention, 1998.

26 Rouse DJ, Stringer JS. An appraisal of screening for maternal type-specific herpes simplex virus antibodies to prevent neonatal herpes. Am J Obstet Gynecol 2000;183:400-6.

27 Qutub M, Klapper P, Vallely P, et al. Genital herpes in pregnancy: is screening cost-effective? Int J STD AIDS 2001;12:14-16.

28 Mindel A, Taylor J, Tideman RL, et al. Neonatal herpes prevention: a minor public health problem in some communities. Sex Transm Infect 2000;76:287-91.

29 Randolph AG, Washington AE, Prober CG. Cesarean delivery for women presenting with genital herpes lesions. Efficacy, risks, and costs [see comments]. JAMA 1993;270:77-82.

30 Scott LL, Sanchez PJ, Jackson GL, et al. Acyclovir suppression to prevent cesarean delivery after first-episode genital herpes. Obstet Gynecol 1996:87:69-73.

31 Centers for Disease Control and Prevention. Notice to Readers National Surveillance for Infectious Diseases, 1995. Morbidity and Mortality Weekly Report 1995;44:737-9 